\title{
The Coadaptation of Parental Supply and Offspring Demand
}

\author{
Mathias Kölliker, ${ }^{1, *}$ Edmund D. Brodie III, ${ }^{1, \dagger}$ and Allen J. Moore ${ }^{2, *}$
}

1. Department of Biology, Indiana University, Bloomington, Indiana 47405;

2. Faculty of Life Sciences, University of Manchester, Oxford Road, Manchester M13 9PT, United Kingdom

Submitted October 13, 2004; Accepted July 5, 2005;

Electronically published August 29, 2005

ABSTRACT: The evolution of parent-offspring interactions for the provisioning of care is usually explained as the phenotypic outcome of resolved conflicting selection pressures. However, parental care and offspring solicitation are expected to have complex patterns of inheritance. Here we present a quantitative genetic model of parentoffspring interactions that allows us to investigate the evolutionary maintenance of a state of resolved conflict. We show that offspring solicitation and parental provisioning are expected to become genetically correlated through coadaptation and that their genetic architecture is dictated by an interaction between patterns of selection and the proximate mechanisms regulating supply and demand. When selection is predominately on offspring solicitation, our model suggests that the genetic correlations between provisioning and solicitation are usually positive if provisioning reduces solicitation. Conversely, when selection is predominately on parental provisioning, the correlations are mostly negative as long as parents show a positive response to offspring demand. Empirical estimates of the genetic architecture of traits involved in family interactions fit these predictions. Our model demonstrates how the evolutionary maintenance of parent-offspring interactions can result in variable patterns of coadaptation, and it provides an explanation for the diversity of family interactions within and among species.

Keywords: parental care, begging, coadaptation, family conflicts, indirect genetic effect, social evolution.

Living in family groups has consequences for both the expression of and selection experienced by diverse phe-

\footnotetext{
* Corresponding author. Present address: Zoological Institute, University of Basel, Evolutionary Biology, Vesalgasse 1, 4051 Basel, Switzerland; e-mail: mathias.koelliker@swissonline.ch.

† E-mail: edb3@bio.indiana.edu.

‡ E-mail: allen.j.moore@manchester.ac.uk.
}

Am. Nat. 2005. Vol. 166, pp. 506-516. (C) 2005 by The University of Chicago. 0003-0147/2005/16604-40679\$15.00. All rights reserved. notypic traits (West-Eberhard 1983; Wolf et al. 1999; Kölliker 2005). Despite the benefits of extended family life, the associated dependence of offspring on parental resources and the cost of this investment to parents lead to potential conflicts among family members. For example, the continuing provisioning of parental resources through parts or all of offspring ontogeny gives rise to the evolution of traits specialized for parent-offspring communication and the regulation of parental provisioning (Alexander 1974; Trivers 1974; Godfray 1995a; Mock and Parker 1997; Wright and Leonard 2002). Simultaneously, such interactions create the possibility for conflicting selection pressures to modify the evolution of these traits and behaviors (Trivers 1974). The optimal level of parental provisioning and offspring demand is predicted to differ among individual family members (Trivers 1974; Godfray 1995a; Mock and Parker 1997).

A considerable number of formal game-theoretic models have been developed to predict the evolutionary resolution of parent-offspring conflict (reviews in Godfray 1995a; Kilner and Johnstone 1997; Mock and Parker 1997; Godfray and Johnstone 2000; Budden and Wright 2001; Parker et al. 2002b; Royle et al. 2002). These treatments usually demonstrate that the phenotypic outcome of this resolution is a combination of costly begging by offspring and parental investment in relation to offspring begging. Conflict resolution is usually modeled as selection on offspring to outcompete siblings for parental resources ("scramble models"; Parker and Macnair 1979; Parker et al. 2002b) or as selection on parents to maximize fitness returns on investment ("honest signaling models"; Godfray 1991; Godfray and Johnstone 2000). Resolution models have proven difficult to test and distinguish experimentally because they often yield common predictions (Mock and Parker 1997; Parker et al. 2002a; Royle et al. 2002). The derivation of testable predictions capable of discriminating between scenarios of conflict resolution is critical to furthering an understanding of the evolution of family interactions (Mock and Parker 1997; Royle et al. 2002, 2004).

A relatively unstudied aspect of the resolution models concerns consequences of a state of resolved conflict for the genetic structure of traits involved in family interac- 
tions (Lynch 1987; Cheverud and Moore 1994; Mock and Parker 1997; Kölliker and Richner 2001; Kölliker 2005). Cheverud (1984), Lynch (1987), Kirkpatrick and Lande (1989), and Cheverud and Moore (1994) were the first to use a quantitative genetic approach to study potential consequences of the genetic architecture of such traits on their evolution. These authors highlight how social traits, which are both genetically variable and have environmental consequences, show complex patterns of inheritance (Wolf et al. 1998; Linksvayer and Wade 2005). In the example of parent-offspring interactions, parents provide to offspring both genes and the environment that influences trait expression (e.g., the level of solicitation), but the parental environment may also vary as a result of heritable differences among parents (Cheverud and Moore 1994). Similarly, offspring represent an environment that may alter parental trait expression (e.g., the level of provisioning) through behaviors such as solicitation (Kölliker 2005). Purely phenotypic approaches fail to capture the genetic complexity that can arise in the evolution of social interactions.

The first quantitative genetic model to explicitly address the genetic architecture of traits involved in parentoffspring interactions incorporated an indirect maternal genetic effect on an offspring trait (Wolf and Brodie 1998). This model showed that in the simple parent-offspring interaction considered, the offspring trait is expected to become coadapted with the maternal performance for this trait. Selectively favored combinations of breeding values for the offspring and parental traits yield, on average, higher fitness than random combinations generating a genetic correlation between them. On the basis of this model, Agrawal et al. (2001) predicted and showed in the burrower bug (Sehirus cinctus) a negative genetic correlation between parental provisioning and offspring elicitation. However, other empirical studies estimated the genetic correlation and found that there may be a positive, negative, or even zero correlation (Kölliker et al. 2000; Kölliker and Richner 2001; Hunt and Simmons 2002; Rauter and Moore 2002; Hager and Johnstone 2003; Curley et al. 2004; Lock et al. 2004; Maestripieri 2004; Kölliker 2005). Thus, the ability to generalize about genetic architecture underlying parent-offspring interactions remains uncertain.

The coadaptation model by Wolf and Brodie (1998) assumes that offspring are passive recipients of parental provisioning and that selection does not impact provisioning. This approach fails to capture much of the dynamic nature of parent-offspring interactions more commonly addressed by optimality models (Kilner and Johnstone 1997; Mock and Parker 1997; Budden and Wright 2001; Royle et al. 2004). Here, we extended the quantitative genetic approach to account for the reciprocal interactions of parent-offspring relations and allow for se- lection to act during both the offspring and the parental life-history stage. We hypothesized that the pattern of coadaptation between offspring solicitation and parental provisioning may reflect whether selection on offspring (analogous to scramble models) or on parents (analogous to honest signaling models) predominates in the evolutionary maintenance of parent-offspring interactions.

\section{A Quantitative Genetic Model of Parent-Offspring Coadaptation}

The modeling approach used here incorporates the interactions of parents and offspring as indirect genetic effects (Moore et al. 1997; Wolf and Brodie 1998; Wolf et al. 1998). The expressions of supply in parents and demand in offspring are modeled as quantitative genetic definitions for linear "effect of supply on demand" and "effect of demand on supply" mechanisms (Mock and Parker 1997; Parker et al. 2002b; Kölliker 2003; Royle et al. 2004). The objective of our study was to model the evolutionary maintenance of a state of resolved conflict when new behavioral variants tend to emerge continuously, for example, through mutation. This perspective differs from conflict resolution models and makes no prediction regarding how the parent-offspring conflict was resolved.

In our model, selection is defined in a quantitative genetic framework as stabilizing selection (Lande and Arnold 1983; Lynch and Walsh 1998; Wolf et al. 1998). Selection gradients define the net relation between relative fitness and (combinations) of trait values and summarize the total fitness cost and benefit schedule (i.e., the partitioning of fitness usually used in resolution models). We use these gradients to describe the average relationship between phenotypes and fitness evaluated at the population mean (Lande and Arnold 1983).

We assume, for simplicity, a single parent cares for a single offspring or batch of noncompeting offspring. Variation in initial offspring condition (Godfray 1991) is not explicitly incorporated but may arise through variation in parental provisioning as mediated by the assumed proximate mechanisms regulating the interaction. We further make the standard assumptions for quantitative genetic models, including adequate additive genetic variation in the traits (i.e., variation based on many loci yielding continuous phenotypic variation and maintained by mutation selection balance), weak selection, random mating, and random environmental deviations that are independent of the additive genetic trait value (Lynch and Walsh 1998; Wolf et al. 1998).

We allow individuals to experience two life-history stages: the offspring stage, during which the solicitation trait is expressed and subject to selection, and a parental stage for those that survive and reproduce, during which 
the provisioning trait is expressed subject to selection. When individuals of the focal generation $t$ are in the offspring stage, they interact with their parents (individuals from the previous generation, $t-1$ ) and experience their provisioning. When these individuals mature and become parents, they interact with their offspring (individuals from the subsequent generation, $t+1)$ and are exposed to solicitation. Our model allows for the evolution of a genetic correlation through the generation of linkage disequilibrium (Tallis and Leppart 1988; Lynch and Walsh 1998; Wolf and Brodie 1998) between solicitation and provisioning loci expressed within a genome. It does not directly consider the effects of pleiotropic mutations or physical linkage that might arise and yield stronger correlations than predicted here. Therefore, the model provides conservative predictions for the genetic correlation (Lande 1984).

\section{Trait Expression}

Let $z_{s}$ be the phenotypic and $a_{s}$ be the additive genetic value for offspring solicitation, and let $z_{p}$ be the expressed phenotypic and $a_{p}$ be the additive genetic value for parental provisioning. Furthermore, $m_{s p}$ is a parental effect coefficient mediating the indirect effect of parental provisioning $(p)$ on offspring solicitation $(s)$, and $o_{p s}$ is an offspring effect coefficient mediating the indirect effect of offspring solicitation on parental provisioning. The phenotypic values for offspring solicitation and parental provisioning can then be defined as $z_{s(t)}=a_{s(t)}+m_{s p} z_{p(t-1)}$ and $z_{p(t)}=$ $a_{p(t)}+o_{p s} z_{s(t+1)}$, respectively. The simultaneous solution to the two recursive equations represents a proximate interaction equilibrium and, thus, the expected expressed levels of $z_{s}$ and $z_{p}$ (Moore et al. 1997; Kölliker 2003):

$$
\begin{aligned}
z_{s(t)} & =\frac{a_{s(t)}+m_{s p} a_{p(t-1)}}{1-m_{s p} o_{p s}}, \\
z_{p(t)} & =\frac{a_{p(t)}+o_{p s} a_{s(t+1)}}{1-m_{s p} o_{p s}} .
\end{aligned}
$$

\section{Fitness and Selection}

Because we are interested in the maintenance of levels of solicitation and parental provisioning reflecting a state of resolved conflict, we assumed that the conflict was resolved constraint-free and that the population mean behaviors are expressed near their ecological optimum (a common assumption in optimality models). Our model addresses the evolutionary maintenance of such an optimum and how it affects the genetic architecture and diversity of parent-offspring interactions. Thus, it is a static coadaptation model and is not designed to provide a solution for the evolutionary dynamics of parent-offspring interactions. Under these conditions, net directional selection can be assumed to be negligible (Gomulkiewicz 1998), and stabilizing selection maintains the parent-offspring interaction near the ecological optimum. Note that this assumption does not require that traits do not experience directional selection during some episodes or life-history stages (Arnold and Wade 1984).

As a consequence, the population means for the traits are taken to remain constant across generations, and only the variances and covariances are allowed to change. The traits $z_{s}$ and $z_{p}$ (as well as their additive genetic values, $a_{s}$ and $a_{p}$, respectively) are defined as deviations from the population mean $\left(z=z_{i}-\bar{z}\right)$, normally distributed with a mean of 0 and variance of 1 . The quadratic regression of total relative fitness on offspring solicitation and parental provisioning $\left(w\left(z_{s}, z_{p}\right)\right)$ is then given by

$$
w\left(z_{s}, z_{p}\right)=\alpha+q_{s} \frac{1}{2} \gamma_{s} z_{s}^{2}+q_{p} \frac{1}{2} \gamma_{p} z_{p}^{2}
$$

where $\alpha$ is a baseline fitness component, $q_{s}$ is the fraction of soliciting individuals in the offspring population, $q_{p}$ is the fraction of provisioning individuals in the parent population, and $\gamma_{s}$ and $\gamma_{p}$ are the quadratic regression coefficients relating relative fitness to solicitation and provisioning, respectively. The values for $\gamma_{s}$ and $\gamma_{p}$ are negative (i.e., selection is stabilizing rather than disruptive). We further assume for simplicity that all offspring solicit and that only one parent cares for the offspring (i.e., either male-only or female-only care). Thus, $q_{s}$ is equal to 1 , and $q_{p}$ is equal to $1 / 2$, with a population sex ratio of unity.

Substituting the trait definitions for $z_{s}$ and $z_{p}$ (eqq. [1]) into equation (2) and expanding yield total fitness as a function of combinations of additive genetic values for solicitation and provisioning:

$$
\begin{gathered}
w\left(z_{s}, z_{p}\right)=\alpha+\frac{1}{2\left(1-m_{s p} o_{p s}\right)^{2}} \\
\times\left(\gamma_{s} a_{s(t)}^{2}+2 \gamma_{s} m_{s p} a_{s(t)} a_{p(t-1)}+\gamma_{s} m_{s p}^{2} a_{p(t-1)}^{2}\right. \\
\left.+\frac{1}{2} \gamma_{p} a_{p(t)}^{2}+\gamma_{p} o_{p s} a_{p(t)} a_{s(t+1)}+\frac{1}{2} \gamma_{p} o_{p s}^{2} a_{s(t+1)}^{2}\right)
\end{gathered}
$$

Because the additive genetic value for a particular trait expressed in a different individual is predictable among interacting family members through their degree of genetic relatedness (Cheverud 1984; Kirkpatrick and Lande 1989; 
Cheverud and Moore 1994; Moore et al. 1997), the subscripts $t-1$ and $t+1$ can be replaced by the coefficient of relatedness $r$ (Cheverud 1984; Cheverud and Moore 1994). This yields the equation for total lifetime fitness in relation to additive genetic values for solicitation and provisioning expressed within an individual over its lifetime:

$$
\begin{gathered}
w\left(a_{s}, a_{p}\right)=\alpha+\frac{1}{2\left(1-m_{s p} o_{p s}\right)^{2}} \\
\times\left(\gamma_{s} a_{s}^{2}+2 r \gamma_{s} m_{s p} a_{s} a_{p}+r^{2} \gamma_{s} m_{s p}^{2} a_{p}^{2}\right. \\
\left.+\frac{1}{2} \gamma_{p} a_{p}^{2}+r \gamma_{p} o_{p s} a_{p} a_{s}+\frac{1}{2} r^{2} \gamma_{p} o_{p s}^{2} a_{s}^{2}\right) .
\end{gathered}
$$

This fitness equation can be translated into an equation describing selection influencing genetic variances and covariances (Lande and Arnold 1983). The parameter $a_{s}^{2}$ corresponds to the direct additive genetic variance in solicitation $G_{s s}, a_{p}^{2}$ corresponds to the direct additive genetic variance in provisioning $G_{p p}$, and $a_{s} a_{p}$ and $a_{p} a_{s}$ correspond to the additive genetic covariances between solicitation and provisioning, $G_{s p}$ and $G_{p s}$ (Wolf and Brodie 1998).

\section{Coadaptation}

The derivation of the genetic correlation among offspring solicitation and parental provisioning requires the extraction of the $\mathbf{G}$ matrix and the matrix of stabilizing selection gradients from equation (4). The $\mathbf{G}$ matrix in our model is defined as

$$
\mathbf{G}=\left(\begin{array}{ll}
G_{s s} & G_{s p} \\
G_{p s} & G_{p p}
\end{array}\right)
$$

Extracting the terms remaining after the $\mathbf{G}$ matrix has been taken into account and arranging them in matrix form give the matrix of selection gradients $\gamma$ (Lande and Arnold 1983; Brodie and Janzen 1996; Wolf and Brodie 1998), which defines the stabilizing selection on offspring solicitation and parental provisioning:

$\gamma=\frac{1}{2\left(1-m_{s p} o_{p s}\right)^{2}}\left(\begin{array}{cc}2 \gamma_{s}+r^{2} o_{p s}^{2} \gamma_{p} & 2 r m_{s p} \gamma_{s}+r o_{p s} \gamma_{p} \\ r o_{p s} \gamma_{p}+2 r m_{s p} \gamma_{s} & 2 \gamma_{p}+2 r^{2} m_{s p}^{2} \gamma_{s}\end{array}\right)$

Coadaptation can then be modeled as an incremental cross-generational change in linkage disequilibrium resulting from selection. The within-generational change in the $\mathbf{G}$ matrix is given by $\Delta \mathbf{G}=\mathbf{G}\left(\gamma-\beta \beta^{\mathrm{T}}\right) \mathbf{G}$ (Lande and
Arnold 1983; Tallis and Leppart 1988; Phillips and Arnold $1989)$, where $\beta$ is the vector of directional selection gradients and the superscript $\mathrm{T}$ denotes matrix transposition. Given our focus on the maintenance of parenting or solicitation at or near an optimum (see above), $\beta$ is set to 0 , yielding $\Delta \mathbf{G}=\mathbf{G} \gamma \mathbf{G}$. By substituting into this equation the matrix of stabilizing selection gradients (eq. [6]) and the $\mathbf{G}$ matrix (eq. [5]) and expanding, we obtain the equations for the within-generational changes in the additive genetic variances $\left(\Delta G_{s s}\right.$ and $\left.\Delta G_{p p}\right)$ as the diagonal elements and covariances $\left(\Delta G_{s p}=\Delta G_{p s}\right)$ as the off-diagonal elements of the resulting matrix:

$$
\begin{gathered}
\Delta G_{s s}=\frac{1}{\left(1-m_{s p} o_{p s}\right)^{2}} \\
\times\left[\gamma_{s}\left(G_{s s}^{2}+2 r m_{s p} G_{s s} G_{s p}+r^{2} m_{s p}^{2} G_{s p}^{2}\right)\right. \\
\left.+\gamma_{p}\left(\frac{1}{2} r^{2} o_{p s}^{2} G_{s s}^{2}+r o_{p s} G_{s s} G_{s p}+G_{s p}^{2}\right)\right] \\
\Delta G_{p p}=\frac{1}{\left(1-m_{s p} o_{p s}\right)^{2}} \\
\times\left[\gamma_{s}\left(r^{2} m_{s p}^{2} G_{p p}^{2}+2 r m_{s p} G_{p p} G_{s p}+G_{s p}^{2}\right)\right. \\
\left.+\gamma_{p}\left(G_{p p}^{2}+r o_{p s} G_{p p} G_{s p}+\frac{1}{2} r^{2} o_{p s}^{2} G_{s p}^{2}\right)\right] \\
\Delta G_{s p}=\frac{1}{\left(1-m_{s p} o_{p s}\right)^{2}} \\
\times\left[\gamma_{s}\left(G_{s p} G_{s s}+r m_{s p} G_{s p}^{2}+r m_{s p} G_{s s} G_{p p}+r^{2} m_{s p}^{2} G_{s p} G_{p p}\right)\right. \\
\left.+\gamma_{p}\left(G_{s p} G_{p p}+\frac{1}{2} r o_{p s} G_{s p}^{2}+\frac{1}{2} r o_{p s} G_{s s} G_{p p}+\frac{1}{2} r^{2} o_{p s}^{2} G_{s p} G_{s s}\right)\right]
\end{gathered}
$$

Note that $\Delta G_{s p}$ is equal to $\Delta G_{p s}$ because we use standardized trait definitions corresponding to $N(0,1)$ for both solicitation and provisioning. Assuming random mating with respect to the genes regulating the expression of parental provisioning and offspring solicitation, the matrix of linkage disequilibrium $\mathbf{D}$ at generation $t+1$ can be defined as $\mathbf{D}_{t+1}=r \mathbf{D}_{t}+r \Delta \mathbf{G}_{t}$ (Tallis and Leppart 1988; Wolf and Brodie 1998). The parameter $\Delta \mathbf{G}_{t}$ is the withingenerational change in the $\mathbf{G}$ matrix during generation $t$ due to stabilizing selection, $\mathbf{D}_{t}$ is the matrix of linkage disequilibrium present before selection in generation $t$, and $r$ is the coefficient of relatedness among parent and offspring. It follows that the $\mathbf{G}$ matrix at generation $t+1$ and before selection is $\mathbf{G}_{t+1}=\mathbf{G}_{0}+\mathbf{D}_{t+1}$, where $\mathbf{G}_{0}$ is the initial G matrix.

We approximated the equilibrium genetic covariances 

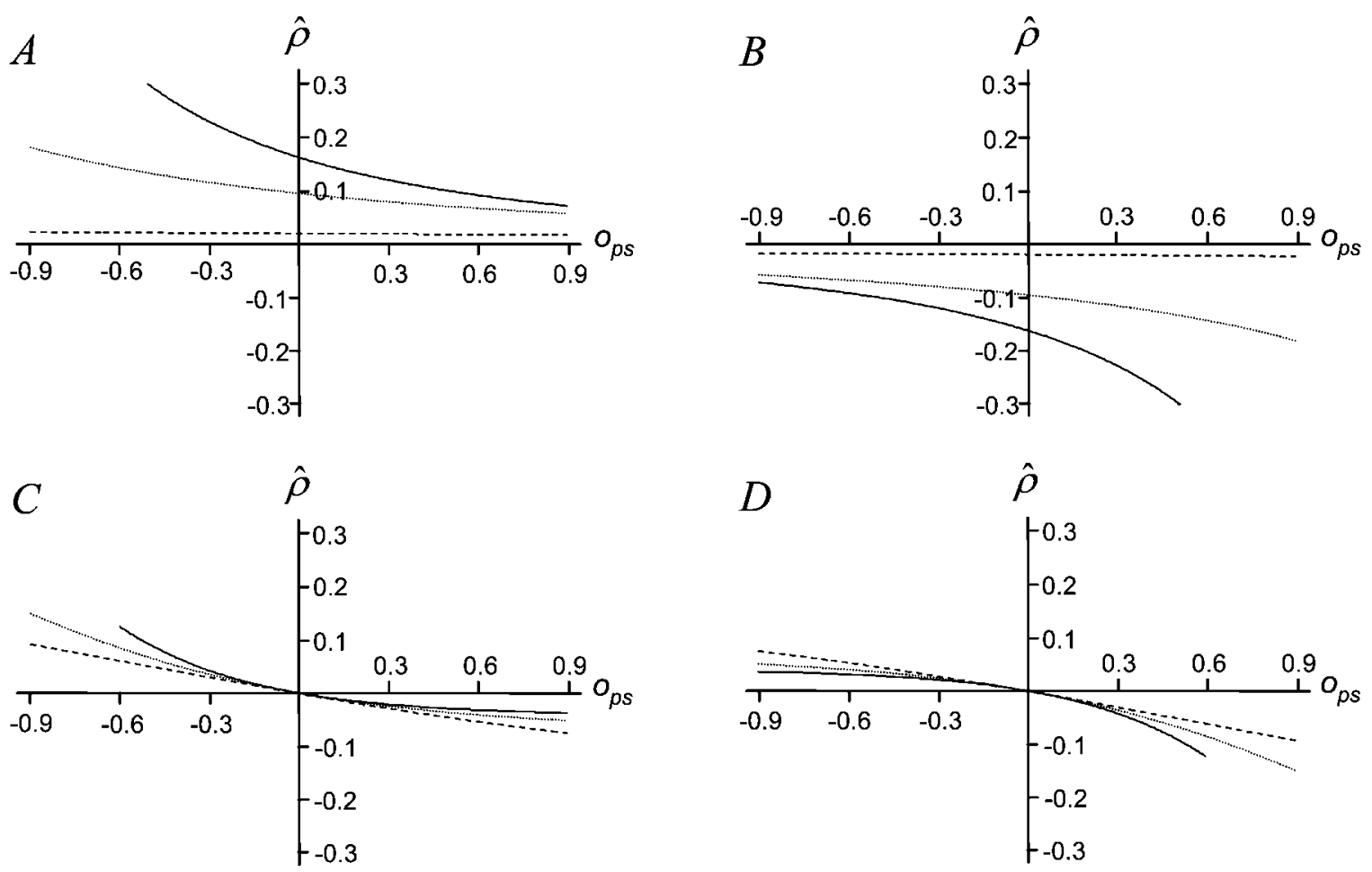

Figure 1: Genetic correlations, $\hat{\rho}$, resulting from stabilizing selection on offspring solicitation $\left(A, B ; \gamma_{s}=-0.7, \gamma_{p}=0\right)$ and parental provisioning $\left(C, D ; \gamma_{s}=0, \gamma_{p}=-0.7\right)$, maintaining provisioning at an intermediate level. The values for the resulting genetic correlation are plotted against $o_{p s}$, the offspring effect coefficient representing a parental response to variation in offspring solicitation. In $A$ and $C$, results for negative values for the parental effect coefficient $m_{s p}$ are shown (solid line, $m_{s p}=-0.9$; dotted line, $m_{s p}=-0.5$; dashed line, $m_{s p}=-0.1$ ). In $B$ and $D$, results for positive values of $m_{s p}$ are shown (solid line, $m_{s p}=0.9$; dotted line, $m_{s p}=0.5$; dashed line, $m_{s p}=0.1$ ).

and variances iteratively (Wolf and Brodie 1998). The initial genetic covariances $G_{s p}$ and $G_{p s}$ were set to 0 . The cumulative change in the $\mathbf{G}$ matrix was simulated over 50 generations. Linkage disequilibrium in all cases stabilized within a few generations. Using 50 generations for the approximation was therefore a sufficiently robust simulation rule. The equilibrium genetic correlation, $\hat{\rho}$, could then be calculated from the equilibrium genetic covariances and variances as $\hat{\rho}=\hat{G}_{s p} /\left(\hat{G}_{p p} \hat{G}_{s s}\right)^{1 / 2}$ (Lynch and Walsh 1998).

\section{Numerical Results of the Model}

Under a first scenario, we assumed that selection on offspring solicitation maintains provisioning at an intermediate level, as expected when selection on offspring to outcompete siblings for parental resources drives coadaptation (analogous to scramble resolution models; Mock and Parker 1997; Parker et al. 2002b). Under a second scenario, stabilizing selection on parental provisioning keeps this trait at an intermediate level of expression, reflecting the maintenance of the parent-offspring interac- tion through selection on parents to maximize fitness returns on investment (analogous to signaling resolution models; Godfray 1991, 1995a, 1995b). These two scenarios represent the two extremes on a power continuum of relative evolutionary control by offspring and their parents (Mock and Parker 1997; Godfray and Johnstone 2000; Parker et al. 2002b). Finally, the generality of the findings from the two scenarios is investigated under simultaneous stabilizing selection on both traits.

\section{Scenario 1: Stabilizing Selection on Offspring Solicitation}

The equations for the within-generational change in the G matrix (eqq. [7]) simplify considerably because the selection gradient for parental provisioning $\gamma_{p}$ is set to 0 . Because of the critical importance of $m_{s p}$ and $o_{p s}$ as parameters defining the proximate nature of the parentoffspring interaction, we investigated coadaptation of offspring solicitation and parental provisioning over a wide range of values for $m_{s p}$ and $o_{p s}$ (fig. $1 A, 1 B$ ). If parental provisioning had a decreasing effect on offspring solicitation $\left(m_{s p}<0\right), \hat{\rho}$ was always positive (fig. $\left.1 A\right)$. As the 
inhibiting effect of provisioning on solicitation becomes stronger (i.e., the more negative $m_{s p}$ ), the value of the positive genetic correlation becomes larger. The offspring effect on provisioning $\left(o_{p s}\right.$; the parental response to solicitation) had under this scenario a modulating effect on the value of $\hat{\rho}$ but did not affect its sign. Conversely, if parental provisioning had an enhancing effect on offspring solicitation $\left(m_{s p}>0\right), \hat{\rho}$ was always negative. The parameter $o_{p s}$ had a modulating effect on the value of $\hat{\rho}$ but not its sign (fig. $1 B$ ).

\section{Scenario 2: Stabilizing Selection on Parental Provisioning}

Proceeding as above, it can be shown that under this second scenario, the sign of the offspring effect coefficient $o_{p s}$ now determines the sign of $\hat{\rho}$ (fig. $1 C, 1 D$ ). If $o_{p s}$ is positive, $\hat{\rho}$ is predicted to be negative and vice versa. This conclusion holds regardless of the value of $m_{s p}$, which affects the value of $\hat{\rho}$ but not its sign (fig. $1 C, 1 D$ ).

\section{Simultaneous Selection on Solicitation and Provisioning}

In reality, most parent-offspring interactions are likely to lie somewhere in between the two scenarios explored above (Godfray and Johnstone 2000; Parker et al. 2002b). This raises the question, what are the general conditions for the evolution of a positive versus negative genetic correlation? We find that the genetic correlation switches sign when the parameter values are such that there is no buildup of linkage disequilibrium from an initial evolutionary stage. The parameter $\Delta G_{s p}$ (eq. [7c]) needs to be equal to 0 after setting $G_{s p}$ to 0 (i.e., no initial covariance):

$$
\frac{1}{\left(1-m_{s p} o_{p s}\right)^{2}}\left(\gamma_{s} r m_{s p} G_{s s} G_{p p}+\gamma_{p} \frac{1}{2} r o_{p s} G_{s s} G_{p p}\right)=0
$$

Rearranging so that all the parameters associated with selection during the offspring stage are on the left-hand side (LHS) and all the parameters associated with selection during the parental stage are on the right-hand side (RHS) of the equation, recalling that we earlier set $q_{s}=1$ and $q_{p}=1 / 2$ and simplifying yield the threshold for the switch from a positive to a negative genetic correlation:

$$
\gamma_{s} q_{s} m_{s p}=-\gamma_{p} q_{p} o_{p s}
$$

If the LHS > RHS, parent-offspring coadaptation generates a positive genetic correlation, and if LHS $<$ RHS, a negative genetic correlation is predicted to evolve.

Comparison of our two scenarios suggests that, with opposing signs for $m_{s p}$ and $o_{p s}$, the sign of $\hat{\rho}$ depends on the patterns of stabilizing selection driving the coadap- tation. Equation (9) allows us to evaluate the generality of this finding with simultaneous selection on both traits. Rearranging this equation and plotting the ratio of selection gradients $\gamma_{s} q_{s} / \gamma_{p} q_{p}$ against the ratio of interaction coefficients $o_{p s} / m_{s p}$ reveals that this generalization holds for $75 \%$ of the parameter space (fig. 2). A positive $\hat{\rho}$ is predicted if selection on solicitation predominates, particularly so if $\left|m_{s p}\right| \geq\left|o_{p s}\right|$ (fig. 2, top right corner). Conversely, a negative $\hat{\rho}$ is predicted if selection on provisioning is stronger, especially if $\left|m_{s p}\right| \leq\left|o_{p s}\right|$ (fig. 2, bottom left corner).

\section{Alignment of the $\mathbf{G}$ Matrix to the Fitness Surface}

When stabilizing selection acts either solely on offspring solicitation or solely on parental provisioning, the fitness surface is characterized by a ridge that reflects combinations of trait values that yield equally high fitness. The genetic correlation is expected to evolve so that it approaches an alignment of trait combinations along that ridge (Blows et al. 2004). We present a numerical example in figure $3 A$ (see also Wolf and Brodie 1998). Although

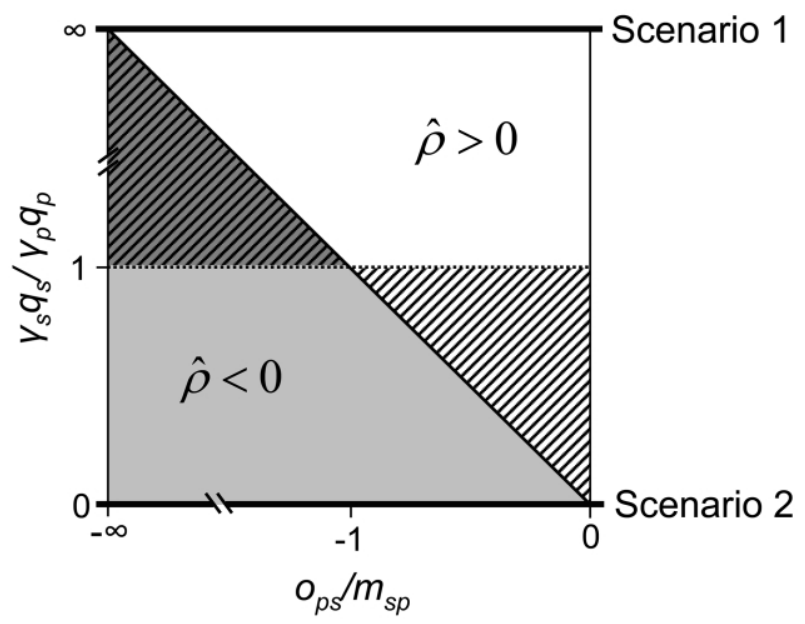

Figure 2: Conditions under which the genetic correlation between solicitation and provisioning, $\hat{\rho}$, reflects patterns of selection maintaining the parent-offspring interaction. The parameter space shown is limited to the range comprising $o_{p s}$ and $m_{s p}$ of opposite sign, and it is plotted as the ratio of stabilizing selection gradients on solicitation and provisioning ( $Y$-axis) against the ratio of the coefficients mediating the parent-offspring interaction, $o_{p s}$ and $m_{s p}$ (X-axis). The diagonal shows where the model predicts $\hat{\rho}$ to be 0 (eq. [9]); $\hat{\rho}$ is predicted to be negative below the diagonal and positive above. The horizontal line depicts the hypothetical separation if the genetic correlation reflects patterns of selection. The dashed areas represent the range under which the sign of $\hat{\rho}$ predicted by the model is the opposite of what would be predicted from patterns of selection alone. The top horizontal line represents our scenario 1 (pure selection on solicitation), and the bottom horizontal line represents our scenario 2 (pure selection on provisioning). 

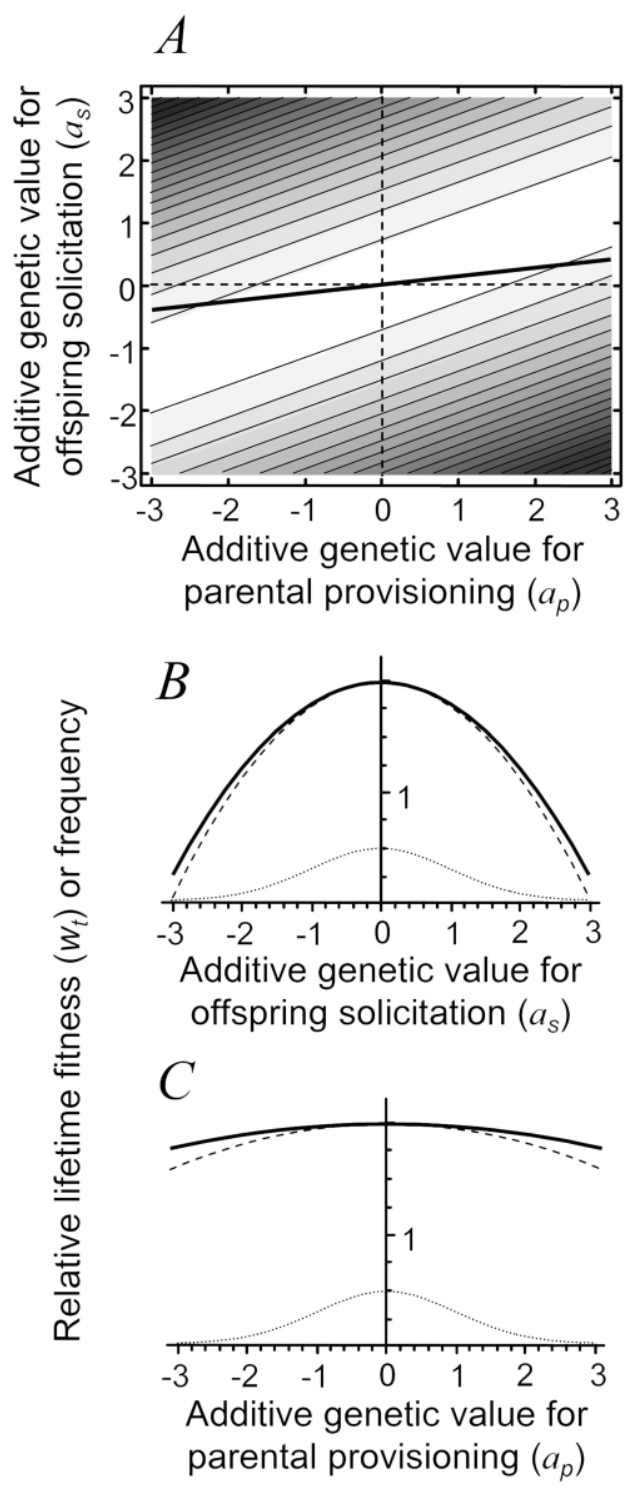

Figure 3: Numerical example of a fitness surface $(A)$ describing relative fitness as a function of all possible combinations of additive genetic values for offspring solicitation and parental provisioning when $\gamma_{s}=-0.7$, $\gamma_{p}=0, m_{s p}=-0.9$, and $o_{p s}=0.3$. Light color indicates high fitness; dark color indicates low fitness. The dashed lines represent average trait combinations in the absence of a genetic correlation and from the perspective of both solicitation (vertical line) and provisioning (horizontal line). The bold solid line stands for trait combinations due to the genetic correlation, $\hat{\rho}$, calculated on the basis of the same parameter values. The individual fitness plots corresponding to the cross sections along these lines are shown from an up-front perspective for $(B)$ offspring solicitation and $(C)$ parental provisioning. In these two figures, solid lines represent fitness functions with the genetic correlation, and the dashed lines represent the fitness function in the absence of a genetic correlation. The dotted lines represent the normal distribution of additive genetic values for solicitation (in $B$ ) and provisioning (in $C$ ). The axes are in units of standard deviations from the trait means. the genetic correlation in our model is always in the direction of the ridge's orientation, the two never fully match under the assumptions of our model. Comparing the individual fitness functions with and without genetic correlation shows that coadaptation nevertheless always leads to higher average fitness of a hypothetical population both during the offspring (fig. $3 \mathrm{~B}$ ) and during the parental stage (fig. 3C). The total beneficial effect of coadaptation on individual fitness over a lifetime corresponds to a combined effect of the two life-history stages. Although figure 3 depicts a numerical example under which a positive genetic correlation evolves, the same conclusion holds for situations favoring negative genetic correlations, that is, that the coadaptation relaxes stabilizing selection on the traits.

\section{Discussion}

Our understanding of the evolution of behavioral interactions has been well informed by investigations of how selection influences phenotypic outcomes, but comparatively little attention has been paid to how the genetic architecture of behavioral traits evolves. Recent theoretical work addressing the evolution of social interactions suggests that a consideration of genetic influences will yield additional insights because social traits are inherited and also act as environments (Moore et al. 1997; Wolf et al. 1998; Kölliker 2005; Linksvayer and Wade 2005). Here, we present the first quantitative genetic model of parental supply and offspring demand incorporating reciprocal parent-offspring interactions as indirect genetic effects (Moore et al. 1997; Wolf et al. 1998). The model shows that patterns of stabilizing selection involved in the evolutionary maintenance of parent-offspring interactions affect the genetic architecture of parental provisioning and offspring solicitation.

Parents, through their provisioning, may be a partially heritable social environmental component to the expression of solicitation (Wolf et al. 1998); offspring, through their solicitation, may act as a partially heritable social environment to the expression of provisioning (Kölliker 2005). If selection acts purely on offspring solicitation, the genetic correlation will reflect offspring adaptation to the genetic variation in the parental provisioning environment. Because only offspring of strongly provisioning parents can afford to express high demand (which under this scenario is costly), a positive correlation is expected. Conversely, if stabilizing selection acts purely on parental provisioning, the correlation reflects parental adaptation to the variation in the offspring solicitation environment. With selection on provisioning, strongly provisioning parents with less demanding offspring fare better because the combination prevents overly costly investment, and the 
high demand of offspring (which under this scenario is not costly) from low-provisioning parents ensures adequate food supply (Wolf and Brodie 1998). This scenario suggests that a negative genetic correlation will evolve.

These verbal arguments are partly confirmed by our model, but the results are more complex, in particular under the more realistic scenario of simultaneous selection on solicitation and provisioning. The sign of the emerging genetic correlation depends on an interaction between the proximate mechanisms regulating the parent-offspring interaction and the relative strengths of stabilizing selection during the offspring versus parental life-history stages. The proximate nature of the parent-offspring interaction was incorporated in our model as a reciprocal feedback interaction (Hussell 1988; Moore et al. 1997; Parker et al. 2002b; Kölliker 2003), where the parental effect coefficient $m_{s p}$ mediates the effect of provisioning on solicitation, and the offspring effect coefficient $o_{p s}$ mediates the effect of solicitation on provisioning (Kölliker 2005). As in previous models (Kirkpatrick and Lande 1989; Moore et al. 1997; Wolf and Brodie 1998), we set these coefficients as fixed model parameters. Allowing $m_{s p}$ and $o_{p s}$ to be part of the evolutionary coadaptation between parental supply and offspring demand adds considerable complexity to the model (Kölliker 2005) and is an interesting future extension.

We performed a broad numerical exploration of our model. Because an overall positive response by parents $\left(o_{p s}>0\right)$ is usual for interactions among begging offspring and provisioning parents, we concentrate our discussion on the model's predictions for positive values of $o_{p s}$. If parental provisioning tends to decrease offspring demand (i.e., $m_{s p}$ is negative), the genetic correlation, $\hat{\rho}$, is predicted to be positive when stabilizing selection acts purely on offspring solicitation (fig. 1A, upper right quadrant) and negative when stabilizing selection acts purely on parental provisioning (fig. $1 C$, lower right quadrant). Such a phenotypic feedback is expected, for example, if parental food provisioning leads to satiation in the offspring, and satiation reduces the intensity of solicitation (Godfray 1991; Parker et al. 2002a). Such hunger/satiation effects on offspring solicitation have been reported widely in birds, mammals, and insects (Weary and Fraser 1995; Kilner and Johnstone 1997; Mock and Parker 1997; Agrawal et al. 2001; Budden and Wright 2001; Royle et al. 2002; Wright and Leonard 2002; Smiseth and Moore 2004), and many systems with parent-offspring interactions involving demand and supply may fall into this range of parameter values of our model (Parker et al. 2002b; Kölliker 2003).

The predictions of our model differ to some extent if the coefficient describing the effect of parental provisioning on offspring solicitation, $m_{s p}$, is positive, for example, if provisioning results in enhanced growth, and larger offspring beg more because they may be more competitive and pay lower begging costs (Mock and Parker 1997; Kilner 2001), or if parents maximize fitness returns on investment by preferentially provisioning high-quality offspring rather than "needy" ones (Cotton et al. 1999). The sign of $\hat{\rho}$ is predicted to be negative under these conditions, irrespective of whether stabilizing selection acts during the offspring (fig. $1 B$, lower right quadrant) or the parental stage (fig. $1 D$, lower right quadrant).

\section{Effects of Coadaptation on Individual Fitness}

The genetic correlation that evolves through coadaptation increases individual mean fitness during both the offspring and the parental stage. If the parameter values are such that a negative $\hat{\rho}$ is favored, the negative genetic correlation relaxes stabilizing selection, and if parameters are such that a positive $\hat{\rho}$ is favored, the positive genetic correlation relaxes stabilizing selection. A few studies using crossfostering experiments show that, as predicted, genetic (coadapted) parent-offspring combinations do better on average than fostered parent-offspring combinations (Hager and Johnstone 2003; Lock et al. 2004).

In our model, $\hat{\rho}$ was generated only through linkage disequilibrium, but this process was not strong enough to allow full alignment of the $\mathbf{G}$ matrix to the ridge of the fitness surface. It is likely that lack of alignment is influenced by our assumption of random mating, which disrupts linkage disequilibrium each generation (Lande 1984; Tallis and Leppart 1988; Wolf and Brodie 1998). Relaxing this assumption by allowing for assortative mating with respect to one or both traits or allowing for some inbreeding, either of which increases the magnitude of covariation among traits and the cross-generational transmission of linkage disequilibrium (Tallis and Leppart 1988), will enhance the buildup of $\hat{\rho}$ (Lande 1984). To illustrate this point, we simulated the genetic correlation under the parameter values used for figure 3 but altering the coefficient of relatedness, $r$, from 0.5 to 0.7 . As a result, the predicted value for $\hat{\rho}$ increased from 0.1 to 0.26 , which represents a quite substantially enhanced alignment to the fitness surface.

Our model does not address how pleiotropic mutations or physical linkage among loci might add to the genetic correlation (Lande 1984). However, pleiotropic mutations affecting the expression of offspring solicitation and parental provisioning simultaneously (or physical linkage among those loci) would be favored if their effects are congruous with the orientation of the fitness surface (Lande 1984). A recent study in mice has demonstrated positive pleiotropic effects of the gene $\mathrm{Peg} 3$ on maternal care behaviors including milk letdown and pup suckling efficiency (Curley et al. 2004). A special class of pleiotropic genes potentially enhancing parent-offspring coadaptation 
is maternal effect genes that are solely expressed in the mother but affect both the mother's provisioning and the offspring's solicitation. The effect on solicitation may be passed on to the offspring through cytoplasmatic components in the oocyte (Wade 1998) and/or hormones (Schwabl and Lipar 2002). A potential example may be the maternal hormones deposited in egg yolk of many bird species (Schwabl and Lipar 2002), although little is known about the covariance between provisioning and begging caused by genetic variation in maternal hormone titers.

\section{The Genetic Correlation as a Testable Signature for Patterns of Selection?}

The predictions of our model suggest that the sign of the genetic correlation under certain conditions reflects patterns of selection involved in the maintenance of parentoffspring interactions. Given that selection can be difficult to measure under field conditions, such an indicator could be very useful to help indirectly infer patterns of selection involved in parent-offspring interactions and to suggest when investigating stabilizing selection might prove informative. Thus, our model provides a testable hypothesis of how selection maintains traits in parent-offspring interactions and influences the genetic architecture underlying such phenotypes.

Because the predicted sign of $\hat{\rho}$ is also critically dependent on $m_{s p}$ and $o_{p s}$, a detailed knowledge of the proximate mechanisms regulating the parent-offspring interaction is required. Currently available empirical estimates of $m_{s p}$ and $o_{p s}$ are often restricted to short-term effects that may be weakened, or even countered, in the long run because of age, body condition, sibling competition, or learning (Price et al. 1996; Kölliker 2003; Wells 2003; Royle et al. 2004). In our model, $m_{s p}$ and $o_{p s}$ are net measures of the effects of parents on offspring and offspring on parents over the total time the parent and its (batch of) offspring interact (Kölliker 2003). A detailed discussion on experimental design for estimating $m_{s p}$ and $o_{p s}$ is provided by Kölliker (2003).

Even with knowledge of the signs of these parameters, there is still $25 \%$ of the parameter space of our model where the sign of $\hat{\rho}$ does not simply reflect selection (fig. 2). The ability to infer patterns of selection based on $\hat{\rho}$ depends on the ratio $o_{p s} / m_{s p}$. With the additional information on the population means for $m_{s p}$ and $o_{p s}$, the likelihood for the sign of the correlation acting as a signature of selection can be increased. If a positive $\hat{\rho}$ co-occurs with an absolute value for $o_{p s}$ equal to or larger than $m_{s p}$, selection on solicitation must dominate coadaptation. Conversely, if a negative $\hat{\rho}$ and $\left|o_{p s}\right| \leq\left|m_{s p}\right|$ are found, $\hat{\rho}$ correctly identifies that selection on provisioning prevails. Experiments that estimate the genetic correlation gain con- siderable interpretational strength by investigating in detail the potentially complex proximate mechanisms regulating parent-offspring interactions.

As in any empirical test of a theoretical model, experimental estimation of the genetic correlation requires controlling for potential confounding factors. In addition to the usual and critical dissection of environmental and genetic components of the covariation (e.g., Lynch and Walsh 1998), the genetic correlation between solicitation and provisioning is potentially confounded by genetic covariation with other traits, for example, clutch size, offspring body condition, or parental body condition (Kölliker et al. 2000; Lock et al. 2004).

\section{Coadaptation and Variation in Life Histories}

We expect stabilizing selection on offspring solicitation to be stronger when brood/littermates compete for parental resources because of the usually greater solicitation costs involved with direct sibling rivalry (Rodríguez-Gironés 1999; Parker et al. 2002a; Royle et al. 2004). Although our model does not explicitly incorporate sibling competition as a factor shaping stabilizing selection on offspring solicitation, it is an unspecified component of the total stabilizing selection gradient $\gamma_{s}$. The (still few) empirical studies that estimate the genetic correlation between solicitation and provisioning fit this expectation and the predictions of our model. In species with the potential for scramble competition through solicitation (i.e., multiple offspring families), including great tits (Parus major, Kölliker et al. 2000), mice (Hager and Johnstone 2003; Curley et al. 2004), and burying beetles (Nicrophorus vespilloides; Lock et al. 2004), positive genetic associations are found. Conversely, in species with very few (often a single) offspring per reproductive attempt, including rhesus macaques (Macaca mulatta; Maestripieri 2004) and sheep (Dwyer and Lawrence 1998), or in species where the parental resource is provided to the clutch as a whole, precluding scramble competition through solicitation (burrower bugs, Sehirus cinctus; Agrawal et al. 2001), negative genetic associations are reported.

On the other hand, stabilizing selection on parental provisioning may depend in part on whether the species is long-lived and iteroparous, where parents must carefully allocate their resources among multiple reproductive attempts to maximize lifetime reproductive success. This trade-off may be less important in short-lived species tending toward semelparity. The prediction for a positive $\hat{\rho}$ would be particularly strong in short-lived species with direct sibling rivalry directed at parents through solicitation. A negative genetic correlation would seem most likely to occur in long-lived species with single offspring per reproductive attempt. 
Additional factors not considered in our model can potentially affect the pattern of coadaptation between solicitation and provisioning. For instance, it has been proposed that a positive genetic correlation might arise because of antagonistic coevolution between the sexes driven by sexual conflict over parental investment (Hager and Johnstone 2003; Royle et al. 2004). Also, the genetic correlation does not necessarily always follow patterns of selection as predicted by our model if pleiotropy or close physical linkage is present. In such cases, genetic correlations are expected to respond slowly to changes in selection patterns (contrary to a genetic correlation due to linkage disequilibrium) and thus may constrain parentoffspring coadaptation at least in the shorter term. Finally, close physical linkage of solicitation, provisioning, or both with other traits could constrain coadaptation and affect the genetic correlation. Nevertheless, our model provides new insights into how patterns of stabilizing selection involved in the maintenance of parent-offspring interactions may shape the genetic architecture of parental supply and offspring demand.

\section{Conclusions}

Current theory for the evolution of family interactions is derived predominately from phenotypic models and emphasizes the evolutionary resolvability of parent-offspring and sibling conflicts (Godfray 1995a; Mock and Parker 1997; Royle et al. 2002). The consequences of continuous regeneration of variation (e.g., through mutation) on the evolutionary maintenance of a state of resolved conflict have not been studied in detail (Cheverud and Moore 1994; Godfray and Johnstone 2000). Our indirect genetic effect model complements current theory by focusing on this aspect and explores the impact of patterns of selection on the genetic variance-covariance structure of solicitation and provisioning traits. It provides a theoretical basis to account for the diversity in family interactions observed within and between species as well as testable hypotheses for the genetics and evolution of social interactions within families.

\section{Acknowledgments}

This study was financially supported by a postdoctoral research fellowship from the Swiss National Science Foundation (M.K.), grants from the National Science Foundation (E.D.B.) and the Natural Environmental Research Council (A.J.M.), and the International Institute for Advanced Study, Nara, Japan, where A.J.M. developed aspects of this model for a symposium on conflict and compromise between parents and offspring. We thank the attendants of the Brodie lab meeting, P. T. Smiseth, J. Wolf, and three anonymous reviewers for discussion and helpful comments.

\section{Literature Cited}

Agrawal, A. F., E. D. Brodie III, and J. Brown. 2001. Parent-offspring coadaptation and the dual genetic control of maternal care. Science 292:1710-1712.

Alexander, R. D. 1974. The evolution of social behavior. Annual Review of Ecology and Systematics 5:325-383.

Arnold, S. J., and M. J. Wade. 1984. On the measurement of natural and sexual selection: theory. Evolution 38:709-719.

Blows, M. W., S. F. Chenoweth, and E. Hine. 2004. Orientation of the genetic variance-covariance matrix and the fitness surface for multiple male sexually selected traits. American Naturalist 163: 329-340.

Brodie, E. D., III, and F. J. Janzen. 1996. On the assignment of fitness values in statistical analyses of selection. Evolution 50:437-442.

Budden, A. E., and J. Wright. 2001. Begging in nestling birds. Current Ornithology 16:83-118.

Cheverud, J. M. 1984. Evolution by kin selection: a quantitative genetic model illustrated by maternal performance in mice. Evolution 38:766-777.

Cheverud, J. M., and A. J. Moore. 1994. Quantitative genetics and the role of the environment provided by relatives in behavioral evolution. Pages 67-100 in C. R. B. Boake, ed. Quantitative genetic studies of behavioral evolution. University of Chicago Press, Chicago.

Cotton, P. A., J. Wright, and A. Kacelnik. 1999. Chick begging strategies in relation to brood hierarchies and hatching asynchrony. American Naturalist 153:412-420.

Curley, J. P., S. Barton, A. Surani, and E. B. Keverne. 2004. Coadaptation in mother and infant regulated by a paternally expressed imprinted gene. Proceedings of the Royal Society of London B 271:1303-1309.

Dwyer, C. M., and A. B. Lawrence. 1998. Variability in the expression of maternal behaviour in primiparous sheep: effects of genotype and litter size. Applied Animal Behaviour Science 58:311-330.

Godfray, H. C. J. 1991. Signalling of need by offspring to their parents. Nature 352:328-330.

. 1995a. Evolutionary theory of parent-offspring conflict. Nature 376:133-138.

- $1995 \mathrm{~b}$. Signaling of need between parents and young: parentoffspring conflict and sibling rivalry. American Naturalist 146:124.

Godfray, H. C. J., and R. A. Johnstone. 2000. Begging and bleating: the evolution of parent-offspring signalling. Philosophical Transactions of the Royal Society of London B 355:1581-1591.

Gomulkiewicz, R. 1998. Game theory, optimization, and quantitative genetics. Pages 283-303 in L. A. Dugatkin and H. K. Reeve, eds. Game theory and animal behavior. Oxford University Press, New York.

Hager, R., and R. A. Johnstone. 2003. The genetic basis of family conflict resolution in mice. Nature 421:533-535.

Hunt, J., and L. W. Simmons. 2002. The genetics of maternal care: direct and indirect genetic effects on phenotype in the dung beetle Onthophagus taurus. Proceedings of the National Academy of Sciences of the USA 99:6828-6832.

Hussell, D. J. T. 1988. Supply and demand in tree swallow broods: 
a model of parent-offspring food-provisioning interactions in birds. American Naturalist 131:175-202.

Kilner, R. 2001. A growth cost of begging in captive canary chicks. Proceedings of the National Academy of Sciences of the USA 98: 11394-11398.

Kilner, R., and R. A. Johnstone. 1997. Begging the question: are offspring solicitation behaviours signals of need? Trends in Ecology \& Evolution 12:11-15.

Kirkpatrick, M., and R. Lande. 1989. The evolution of maternal characters. Evolution 43:485-503.

Kölliker, M. 2003. Estimating mechanisms and equilibria for offspring begging and parental provisioning. Proceedings of the Royal Society of London B 270(suppl.):S110-S113.

. 2005. Ontogeny in the family. Behavior Genetics 35:7-18.

Kölliker, M., and H. Richner. 2001. Parent-offspring conflict and the genetics of offspring solicitation and parental response. Animal Behaviour 62:395-407.

Kölliker, M., M. W. G. Brinkhof, P. Heeb, P. Fitze, and H. Richner. 2000. The quantitative genetic basis of offspring solicitation and parental response in a passerine bird with biparental care. Proceedings of the Royal Society of London B 267:2127-2132.

Lande, R. 1984. The genetic correlation between characters maintained by selection, linkage and inbreeding. Genetical Research 44: 309-320.

Lande, R., and S. J. Arnold. 1983. The measurement of selection on correlated characters. Evolution 37:1210-1226.

Linksvayer, T., and M. J. Wade. 2005. The evolutionary origin and elaboration of sociality in the aculeate hymenoptera: maternal effects, sib-social effects, and heterochrony. Quarterly Review of Biology (forthcoming).

Lock, J. E., P. T. Smiseth, and A. J. Moore. 2004. Selection, inheritance, and the evolution of parent-offspring interactions. American Naturalist 164:13-24.

Lynch, M. 1987. Evolution of intrafamilial interactions. Proceedings of the National Academy of Sciences of the USA 84:8507-8511.

Lynch, M., and B. Walsh. 1998. Genetics and analysis of quantitative traits. Sinauer, Sunderland, MA.

Maestripieri, D. 2004. Genetic aspects of mother-offspring conflict in rhesus macaques. Behavioral Ecology and Sociobiology 55:381387.

Mock, D. W., and G. A. Parker. 1997. The evolution of sibling rivalry. Oxford Series in Ecology and Evolution. Oxford University Press, Oxford.

Moore, A. J., E. D. Brodie III, and J. B. Wolf. 1997. Interacting phenotypes and the evolutionary process. I. Direct and indirect genetic effects of social interactions. Evolution 51:1352-1362.

Parker, G. A., and M. R. Macnair. 1979. Models of parent-offspring conflict. IV. Suppression: evolutionary retaliation by the parent. Animal Behaviour 27:1210-1235.

Parker, G. A., N. J. Royle, and I. R. Hartley. 2002a. Begging scrambles with unequal chicks: interactions between need and competitive ability. Ecology Letters 5:206-215.

. 2002b. Intrafamilial conflict and parental investment: a synthesis. Philosophical Transactions of the Royal Society of London B 357:295-307.
Phillips, P. C., and S. J. Arnold. 1989. Visualizing multivariate selection. Evolution 43:1209-1222.

Price, K., H. Harvey, and R. Ydenberg. 1996. Begging tactics of nestling yellow-headed blackbirds, Xanthocephalus xanthocephalus, in relation to need. Animal Behaviour 51:421-435.

Rauter, C. M., and A. J. Moore. 2002. Evolutionary importance of parental care performance, food resources, and direct and indirect genetic effects in a burying beetle. Journal of Evolutionary Biology 15:407-417.

Rodríguez-Gironés, M. A. 1999. Sibling competition stabilizes signalling resolution models of parent-offspring conflict. Proceedings of the Royal Society of London B 266:2399-2402.

Royle, N. J., I. R. Hartley, and G. A. Parker. 2002. Begging for control: when are offspring solicitation behaviours honest? Trends in Ecology \& Evolution 17:434-440.

- 2004. Parental investment and family dynamics: interactions between theory and empirical tests. Population Ecology 46:231241.

Schwabl, H., and J. Lipar. 2002. Hormonal regulation of begging behaviour. Pages 221-244 in J. Wright and M. L. Leonard, eds. The evolution of begging: competition, cooperation and communication. Kluwer Academic, Dordrecht.

Smiseth, P. T., and A. J. Moore. 2004. Signalling of hunger when offspring forage by both begging and self-feeding. Animal Behaviour 67:1083-1088.

Tallis, G. M., and P. Leppart. 1988. The joint effects of selection and assortative mating on multiple polygenic characters. Theoretical and Applied Genetics 75:278-281.

Trivers, R. L. 1974. Parent-offspring conflict. American Zoologist 14: 249-264.

Wade, M. J. 1998. The evolutionary genetics of maternal effects. Pages 5-21 in T. A. Mousseau and C. W. Fox, eds. Maternal effects as adaptations. Oxford University Press, New York.

Weary, D. M., and D. Fraser. 1995. Calling by domestic piglets: reliable signals of need? Animal Behaviour 50:1047-1055.

Wells, J. C. K. 2003. Parent-offspring conflict theory, signaling of need, and weight gain in early life. Quarterly Review of Biology 78:169-202.

West-Eberhard, M. J. 1983. Sexual selection, social competition, and speciation. Quarterly Review of Biology 58:155-183.

Wolf, J. B., and E. D. Brodie III. 1998. The coadaptation of parental and offspring characters. Evolution 52:299-308.

Wolf, J. B., E. D. Brodie III, J. M. Cheverud, A. J. Moore, and M. J. Wade. 1998. Evolutionary consequences of indirect genetic effects. Trends in Ecology \& Evolution 13:64-69.

Wolf, J. B., E. D. Brodie III, and A. J. Moore. 1999. Interacting phenotypes and the evolutionary process. II. Selection resulting from social interactions. American Naturalist 153:254-266.

Wright, J., and M. L. Leonard. 2002. The evolution of begging: competition, cooperation and communication. Kluwer Academic, Dordrecht.
Associate Editor: Mark W. Blows Editor: Michael C. Whitlock 